\title{
Attenuation by contextual cues of retroactive interference of a conditional discrimination in rats
}

\author{
WARD A. RODRIGUEZ, LOUIS S. BORBELY, and RONALD S. GARCIA \\ New Mexico Highlands University, Las Vegas, New Mexico
}

\begin{abstract}
We investigated the effect of associating unique contextual cues with an interpolated learning task on retroactive interference in long-term memory. Rats were originally trained in a two-bar operant chamber with an auditory conditional discrimination stimulus. During interpolated learning, which occurred in either the original or a new context, some rats were trained on a probability learning task that did not include the auditory stimuli present during original learning. Subsequent retraining on the original conditional discrimination task in the original context showed that (1) significant retroactive interference occurs in rats, and (2) the presence of unique contextual cues during interpolated learning significantly reduces this interference. These results extend the conditions under which the susceptibility to retroactive interference can be altered by contextual cues.
\end{abstract}

The term retroactive interference is used to describe both an experimental paradigm and an empirical phenomenon. The standard research design for studying retroactive interference includes an experimental group that receives original learning, interpolated learning, and a test for the retention of the original learning, and a control group that receives original learning and testing but does not receive any interpolated learning. These experimental operations provide empirical evidence for interference-that is, detrimental effects on the retention of the original learning as a consequence of the interpolated learning activity.

Prior to the adoption of an information processing paradigm, retroactive interference was studied extensively in humans (for reviews of method and theory, see Keppel, 1968; Postman, 1971; Postman \& Underwood, 1973). Although the study of retroactive interference in humans continues to be of interest (Bjork \& Richardson-Klavehn, 1989), it is not as central as it once was. In contrast, the study of retroactive interference in animals has increased as a result of the willingness of animal researchers to employ a more cognitive analysis. In most of the studies with humans, researchers have examined the effects of retroactive interference on long-term memory; most animal studies have been focused on the effects of retroactive interference on short-term memory (Grant \& Roberts, 1976; Kendrick \& Rilling, 1986; Medin, Reynolds, \& Parkinson, 1980; Roitblat, 1987).

This research was supported by National Institute of General Medical Sciences MBRS Grant S06 GM08066-20 to W.A.R. and by a Supplement for Underrepresented Minorities in Biomedical Research awarded to W.A.R. in conjunction with NIDA Grant DA06192 to Joe L. Martinez, Jr. We thank Kathy Wilmington and Susan B. Rodriguez for their comments and assistance. Requests for reprints should be addressed to W. A. Rodriguez, Department of Psychology, 3210 Tolman Hall, University of California, Berkeley, CA 94720.
In investigations of the effects of retroactive interference on long-term memory in animals, the same set of stimuli and responses has typically been used during both the original learning task and the interpolated learning task. In these studies, the relationship between stimuli and the response-outcome contingencies have differed (Wickens, Tuber, Nield, \& Wickens, 1977) or have been reversed (Chiszar \& Spear, 1969; Crowder, 1967; Zentall, 1970) in the two training conditions. In contrast, Heinemann, Sage-Day, and Brenner (1981) reported that retroactive interference in long-term memory can be observed when the interpolated task does not involve the same discriminative stimuli as have been present during the original learning. In their study, all pigeons originally learned a conditional discrimination. In one variation of the task, discriminable levels of white noise set the occasion, or were conditional cues, for signaling which of two response keys could be pecked for reinforcement. Terminal interpolated learning consisted of a two-key probability learning (20\%) task; the conditional cues present during original learning were not present during interpolated learning. When compared with the performance of pigeons that had remained in their home cages, the performance of pigeons receiving the interpolated probability learning task was reduced after reinstatement of the original conditional discrimination. However, because the conditional discriminative cues present during the original learning were absent during the interpolated learning, the source of the interference in the Heinemann et al. study is not obvious.

The purpose in the present experiment was to investigate factors that might influence the retroactive interference observed by Heinemann et al. (1981). Our approach was to determine whether we could alleviate or attenuate retroactive interference by using experimental manipula- 
tions that have been shown in other studies to affect the magnitude of retroactive interference in humans and animals. Specifically, we examined the effects of changing both the environmental context and the conditions of reward associated with the interpolated task on the performance of the reinstated original learning task.

Acquiring the original and interpolated learning tasks in the presence of unique contextual cues attenuates retroactive interference effects on long-term memory in humans (Bilodeau \& Schlosberg, 1951; Greenspoon \& Ranyard, 1957; Strand, 1970) and in rats (Bouton \& Swartzentruber, 1989; Zentall, 1970). Contextual cues may be an especially important source of interference in animal studies (Wickens, 1987). Animals often perform according to the innate eprior doctrine (Staddon, 1983), appearing to be guided most strongly by what they have learned most recently. Thus, when both original and interpolated learning occur in the same context, the simultaneous activation of two disparate sets of contingencies may disrupt performance during testing. Spear (1981) and others (Estes, 1973; Medin, 1975) have suggested that, in animals, contextual cues may play such a role during memory retrieval.

The conditions of reward during an interpolated learning task can have major effects on transfer and transitional behavior (Logan \& Ferraro, 1978). Thus the amount of retroactive interference is reduced when each discrimination is learned under different incentive conditions (Glendenning \& Meyer, 1971). Presenting qualitatively different rewards during the original and interpolated learning phases may aid the animals in differentiating the two learning tasks (Peterson, Wheeler, \& Armstrong, 1978; Trapold, 1970) and thereby reduce retroactive interference. Following this logic, in addition to changing the experimental context during interpolated learning, we also changed the quality of reinforcement during this phase.

Finally, the conditional discrimination and the probability learning task used by Heinemann et al. (1981) resulted in a difference in the number of rewarded responses during the original learning ( 80 rewards) and the probability learning ( 20 rewards) sessions. When there are large changes between the number of reinforcers received during original and interpolated learning, it is unclear to what extent the retroactive interference effects are due to changes in the probability of reward or to other differences between the two tasks. In the present study, we examined the possible effect of differences in reward probability by including two probability learning conditions: a probability learning $50 \%$ group and a probability learning $90 \%$ group. The latter more closely matched the probability of reward received during the original conditional discrimination (approximately $87 \%$ ).

\section{METHOD}

\section{Subjects}

The subjects were 98 experimentally naive male rats of the Sprague-Dawley strain. All rats were approximately 90 days of age at the beginning of the experiment and were housed individually in a temperature-controlled room $\left(23^{\circ} \pm 1^{\circ} \mathrm{C}\right)$. The experiment was conducted at the same time each day during the light phase of a 12:12-h light:dark cycle (lights on at 7:00 a.m.). During the experiment, the rats had free access to water, but the availability of food was controlled to maintain the rats at $80 \%$ of their free-feeding body weights.

\section{Apparatus}

The apparatus consisted of two identical Coulbourn Instruments two-lever rat operant conditioning chambers. Each modular test chamber was equipped with a recessed food magazine in the center of the end wall and a retractable bar on each side of the magazine. Either solid (45-mg Noyes pellets) or liquid $(0.10 \mathrm{ml}$ of Borden Kitten Milk Replacer) food reinforcers could be delivered into the magazine. Extensive work in our laboratory has shown that these two rewards, although qualitatively different, produce and maintain equivalent levels of performance. Mounted on the end wall were a speaker that permitted the presentation of an $1800-\mathrm{Hz}$ tone, and a click generator with which a $20-\mathrm{Hz}$ train of clicks could be presented. The stimuli, delivered by both the speaker and the click generator, measured approximately $76 \mathrm{~dB}$ (re: $20 \mu \mathrm{N} / \mathrm{m}^{2}$; A scale), and ventilation fans provided a background noise level of $62 \mathrm{~dB}$ (A), as measured close to the food magazine. Each conditioning chamber was enclosed in its own sound-and light-attenuating shell. Experimental control and recording were accomplished with the use of solid state modules.

Training occurred in one of two experimental contexts. The original context $(O C)$ consisted of an experimental chamber measuring $29.5 \times 24 \times 29 \mathrm{~cm}$. The floor of the chamber was composed of 0.5 -cm stainless steel rods, spaced $1.0 \mathrm{~cm}$ apart. The two end walls and the top of the chamber were aluminum; the sidewalls were clear Plexiglas. A 7.5-W white houselight, which was mounted on the rear wall of the shell, provided constant indirect illumination. No distinct olfactory cues were present in the chamber pan.

The new context (NC) was produced by modifying a number of the characteristics of the original context. The houselight was turned off, cedar chips were placed in the chamber pan, the texture of the floor was modified by insertion of a 2-mm square wire mesh, and the length of the chamber was reduced to $12 \mathrm{~cm}$ by the insertion of a black panel into the rear of the chamber.

\section{Procedure}

Preliminary training. Preliminary training consisted of training the animals in the original context to barpress for solid and liquid reward. Only one response bar was inserted at a time into the chamber during preliminary training. Whether the animal was initially trained on the right or the left bar was randomly determined. All animals were trained initially to press the bar in order to receive food pellets. During the first daily session, food was delivered on a variable 1-min schedule; in addition, each barpress produced a pellet. Upon completion of 50 barpresses, the session was terminated. Identical variable-time and response-contingent schedules of training for liquid food reward with the other response bar occurred during the second daily session.

The remainder of the experiment consisted of three phases: a conditional discrimination learning phase, an interpolated learning phase, and a phase of retraining on the original conditional discrimination. During all of these phases, daily sessions consisted of 80 trials separated by intertrial intervals of $10 \mathrm{sec}$. Thirty consecutive training sessions were given during each phase.

Phase 1: Conditional discrimination training. Animals were trained on a conditional discrimination task in the original experimental context. Each conditional discrimination training trial began with the presentation of either a tone or a click. The order of stimulus presentation was random. A barpress on one of the two levers was considered a correct response if it occurred during presentation of one of the two auditory stimuli, and a barpress on the 
Table 1

Interpolated Training

\begin{tabular}{cc}
\hline Group & Sample Size \\
\hline PL50\%/OC/S & 14 \\
PL50\%/OC/L & 14 \\
PL50\%/NC/S & 13 \\
PL50\%/NC/L & 13 \\
PL90\%/OC/L & 12 \\
CD/NC/S & 12 \\
NIL & 15
\end{tabular}

Note-PL, probability learning; OC, original context; NC, new context; $S$, solid reinforcer; $L$, liquid reinforcer; $C D$, conditional discrimination learning; NIL, no interpolated learning.

other bar was considered a correct response if it occurred during presentation of the other auditory stimulus. A barpress on either lever terminated the auditory stimulus and retracted both levers for $10 \mathrm{sec}$. Correct choices resulted in delivery of a liquid food reward. After an incorrect choice, the trial was repeated (i.e., a correction procedure was employed).

Phase 2: Interpolated training. Animals were assigned randomly to one of seven interpolated learning conditions. Table I displays how the groups varied according to learning task, training context, and reward condition. Four different learning conditions were used: probability learning (PL) $50 \%$ or $90 \%$, in which two response bars were inserted into the chamber in the absence of auditory discriminative stimuli and responses on either bar were reinforced on $50 \%$ or $90 \%$ of the trials; conditional discrimination learning (CD), which was a continuation of the Phase 1 training; and no interpolated learning (NIL), in which the animals remained in their home cages except for weighing and daily handling. Training was conducted in either the original context (OC) or the new context (NC), as described above. During interpolated training, the animals were rewarded with either solid (S) or liquid (L) reinforcers. Table 1 also displays the sample size for each group.

Phase 3: Retraining conditional discrimination. All the animals were retrained in the original experimental context on the conditional discrimination task with liquid reward.

\section{RESULTS}

All data were subjected to a number of exploratory data analytic techniques (Hoaglin, Mosteller, \& Tukey, 1991) in order to ensure that the analysis of variance model tested was appropriate for the data. In order to ensure that the assumptions of the analysis of variance were met, the proportions of correct responses were subjected to an arcsine transformation prior to inferential statistical testing. An analysis of variance of the mean proportion of correct responses for each group during the last 5 days of the original conditional discrimination showed that all groups reached similar asymptotes $[M=.96, F(6,91)=$ $1.15, p>.25$ ], that there was no effect of days $(F<$ $1.0)$, and that there was no significant group $\times$ day interaction $[F(24,364)=1.21, p>.20]$. An additional analysis was conducted to determine whether the animals acquired the original conditional discrimination task at similar rates. This analysis was based on the time at which each rat's performance reached an asymptotic level. The asymptote was defined as the session number on which each animal had performed for 2 consecutive days above the level of its median performance during the last 5 days of the phase. The analysis indicated that the rate of acquisition did not differ among the groups $[M=14.7$; $F(6,91)=1.43, p>.20$ ]. Together, these results show that all groups had similar rates of acquisition, and that they reached a common and stable asymptote during the original conditional discrimination learning task.

The mean proportion of reinforced trials during the last 5 days of interpolated training was also analyzed. A one-way analysis of variance showed that the interpolated performances of all four PL50\% groups did not differ significantly $(F<1.0)$. However, the mean proportion of reinforced responses for group $\mathrm{CD} / \mathrm{NC} / \mathrm{S}(M=.97)$ was greater than the expected performance of .90 reinforced responses for Group PL90\%/OC/L $[t(11)=27.10, p<.001]$. Thus, our attempts to equate the number of reinforcements received by Groups PL $90 \% / O C / L$ and $\mathrm{CD} / \mathrm{NC} / \mathrm{S}$ during interpolated learning was not achieved.

The performance of group $\mathrm{CD} / \mathrm{NC} / \mathrm{S}$ on the 1st day in the new context was reduced by .15 from the original learning baseline. This decrease in performance was statistically significant $[F(1,11)=50.69, p<.001]$, indicating that a shift to a different context and a qualitatively different reinforcer initially disrupted performance of the conditional discrimination task. However, this disruption in performance was temporary because there were no statistically significant differences in the mean proportion of correct responses made at asymptote during the original learning baseline and the interpolated learning baseline for Group CD/NC/S $(F<1.0)$. This indicates that the original and new contexts were able to support equivalent conditional discrimination performance.

Figure 1 depicts the mean proportion of correct responses made by each group on the 1st day on which the original conditional discrimination task was reintroduced in the original training context. Because there were no differences in performance between animals that had been rewarded with liquid or solid rewards during the interpolated task, we combined these groups in Figure 1 as well as in the statistical analyses. Interpolated PL50\% training in either the original or the new context disrupted performance on the 1st day of the reinstated conditional discrimination learning task, relative to the performance of the NIL control group $[O C, F(1,41)=27.89, p<.001$; NC, $F(1,39)=23.13, p<.001]$. More importantly, the disruption in performance was greater in the animals receiving the interpolated PL50\% in the original context than in the animals receiving PL50\% interpolated training in the new context $[F(1,52)=6.06, p<.02]$. Thus, interpolated training in a discriminably different context produced less interference than did interpolated training in the same context as that in which the original conditional discrimination learning task was presented.

Figure 1 also shows that PL90\%/OC/L interpolated training resulted in the poorest performance when the original conditional discrimination contingencies were reinstated. This decrement in performance was significantly different from the performance of the NIL control group $[F(1,25)=$ $18.82, p<.0011$. However, comparison of the mean per- 


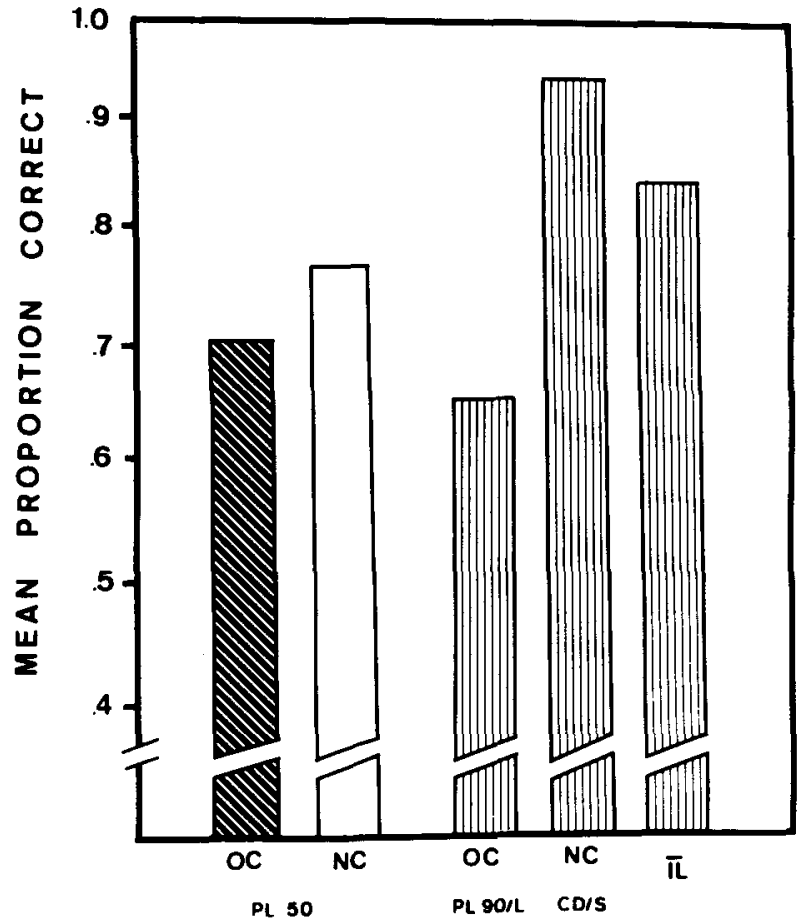

Figure 1. Mean proportion of correct (reinforced) responses for each interpolated learning group on Day 1 of Phase 3: Retraining on the conditional discrimination. Group abbreviations are explained in Table 1.

formance of the two groups receiving PL50\% in the original context with the mean performance of the animals receiving PL90\% showed that the two probability learning conditions did not produce significantly different performance $[F(1,38)=1.60, p>.21]$.

An analysis of the mean proportion of reinforced responses made by each group during the last 5 days of the reinstated conditional discrimination task showed that all groups reached a similar asymptote $(F<1.0)$. However, Group $\mathrm{CD} / \mathrm{NC} / \mathrm{S}$ relearned faster than did the other groups $[F(1,91)=5.37, p<.023]$. Stable conditional discrimination performance was reached faster after interpolated learning ( $M=7.4$ days) than it was during original learning $[M=14.7$ days; $F(6,91)=3.41, p<.025]$.

\section{DISCUSSION}

In the present experiment, we showed that retroactive interference with a conditional discrimination in rats occurs even when the interpolated task does not contain conditional cues relevant to solving the original conditional learning task. These results are similar to those found by Heinemann et al. (1981) using pigeons as subjects. Both the amount of retroactive interference produced in the original context and the asymptotic level of performance during the conditional discrimination task were nearly identical in the two studies. The major difference in the performance of our rats versus that of the pigeons studied by Heinemann et al. occurred during relearning of the conditional discrimination. Although reacquisition was gradual in both studies, reacquisition of the conditional discrimination in the present study was faster than was original learning. Heinemann et al. reported nearly identical original and relearning acquisition curves. Bitterman (1969) has argued, and we observed, that rats, unlike pigeons, tend to respond almost exclusively to one of the two response bars during a PL50\% task. Perhaps differences in response tendencies during interpolated PL50\% in the present study and during PL20\% in the Heinemann et al. study can account for some of the differences in the relearning of the original conditional discrimination.

Interpolated PL50\% and PL90\% training both resulted in extensive retroactive interference. These results extend the conditions under which retroactive interference can be observed to include interpolated learning occurring in the absence of cues relevant to original learning. On the other hand, the quality of reward received during interpolated learning did not affect retroactive interference.

Most importantly, in the present experiment we were able to attenuate the amount of retroactive interference by correlating unique contextual cues with the original and the interpolated tasks. Our results suggest that at least part of the interference in the Heinemann et al. (1981) study was due to the presence of identical contextual cues during original learning and interpolated learning. Our results are similar to those of Zentall (1970), who also showed that changes in context during interpolated reversal training of a spatial discrimination task attenuate forgetting.

Contextual cues are believed to facilitate the retrieval of memories in animals (Bouton \& Swartzentruber, 1989; Estes, 1973; Medin, 1975; Spear, 1981; Wickens, 1987). These cues may serve to tag or discriminate two accessible memories and thereby reduce interference produced by conflicting memories. The results of the present experiment support this hypothesis.

In summary, in the present experiment we demonstrated that significant retroactive interference with an auditory conditional discrimination task can occur in rats even when the interpolated learning task does not contain the original conditional discriminative stimuli. Furthermore, the degree of interference can be attenuated if each task is learned in a distinctive environmental context. These data accord with the suggestion that retrieval can be a major source of interference in animal learning.

\section{REFERENCES}

Bilodeau, I. M., \& Schlosberg, H. (1951). Similarity in stimulating conditions as a variable in retroactive inhibition. Joumal of Experimental Psychology, 41, 199-204.

Bitterman, M. E. (1969). Habit-reversal and probability learning: Rats, birds, and fish. In R. M. Gilbert \& N. S. Sutherland (Eds.), Animal discrimination learning (pp. 163-175). New York: Academic Press.

Bjork, R. A., Richardson-Klavehn, A. (1989). On the puzzling relationship between environmental context and human memory. In C. Lzawa (Ed.), Current issues in cognitive processes: The Tulane Flowerree Symposium on Cognition (pp. 313-344). Hillsdale, NJ: Erlbaum. 
Bouton, M. E., \& SWArtzentruber, D. (1989). Slow reacquisition following extinction: Context, encoding, and retrieval mechanisms. Journal of Experimental Psychology: Animal Behavior Processes, 15, 43-53.

Chiszar, D. A., \& Spear, N. E. (1969). Stimulus change, reversal learning, and retention in the rat. Journal of Comparative \& Physiological Psychology, 69, 190-195.

Crowder, R. G. (1967). Proactive and retroactive inhibition in the retention of a T-maze habit in rats. Journal of Experimental Psychology, 74, $167-171$.

Estes, W. K. (1973). Memory and conditioning. In F. J. McGuigan \& D. B. Lumsden (Eds.), Contemporary approaches to conditioning and learning (pp. 265-280). New York: Wiley.

Glendenning, R. L., Meyer, D. R. (1971). Motivationally related retroactive interference in discrimination learning by rats. Journal of Comparative \& Physiological Psychology, 75, 153-156.

Grant, D. S., RoberTs, W. A. (1976). Sources of retroactive inhibition in pigeon short-term memory. Journal of Experimental Psychology: Animal Behavior Processes, 2, 1-16.

GreENSPOON, J., RaNyard, R. (1957). Stimulus conditions and retroactive inhibition. Joumal of Experimental Psychology, 53, 55-59.

Heinemann, E. G., Sage-Day, J., \& Brenner, N. (1981). Retroactive interference in discrimination learning. Science, 214, 1254-1257.

Hongun, D. C., Mosteller, F., Tukey, J. W. (1991). Fundamentals of exploratory analysis of variance. New York: Wiley.

Kendrick, D. F., \& Rilling, M. E. (1986). AIM: A theory of active and inactive memory. In D. F. Kendrick, M. E. Rilling, \& M. R. Denny (Eds.), Theories of animal memory (pp. 129-152). Hillsdale, NJ: Erlbaum.

KEPPEL, G. (1968). Retroactive and proactive inhibition. In T. R. Dixon \& D. L. Horton (Eds.), Verbal behavior and general behavior theory (pp. 172-213). Englewood Cliffs, NJ: Prentice-Hall.

Logan, F. A., \& Ferraro, D. P. (1978). Systematic analyses of leaming and motivation. New York: Wiley.

Medin, D. L. (1975). A theory of context in discrimination learning. In G. H. Bower (Ed.), The psychology of learning and motivation (Vol. 9, pp. 263-314). New York: Academic Press.

Medin, D. L., Reynolds, T. J., \& PARKinson, J. K. (1980). Stimulus similarity and retroactive interference and facilitation in monkey short-term memory. Journal of Experimental Psychology: Animal Behavior Processes, 6, 112-125.

Peterson, G. B., Wheeler, R. L., A Armstrong, G. D. (1978). Expectancies as mediators in the differential-reward conditional discrimination performance of pigeons. Animal Learning \& Behavior, 6 , 279-285.

Postman, L. (1971). Transfer, interference and forgetting. In J, W. Kling \& L. A. Riggs (Eds.), Woodworth and Schlosberg's Experimental psychology (3rd. ed., pp. 1019-1132). New York: Holt, Rinehart \& Winston.

Postman, L., \& Underwood, B. J. (1973). Critical issues in interference theory. Memory \& Cognition, 1, 19-40.

Rortblat, H. L. (1987). Introduction to comparative cognition. New York: W. H. Freeman.

SPEAR, N. E. (1981). Extending the domain of memory retrieval. In N. E. Spear \& R. R. Miller (Eds.), Information processing in animals: Memory mechanisms (pp. 341-378). Hillsdale, NJ: Erlbaum. STADDON, J. E. R. (1983). Adaptive behavior and leaming. New York: Cambridge University Press.

Strand, B. Z. (1970). Change of context and retroactive inhibition. Journal of Verbal Learning \& Verbal Behavior, 9, 202-206.

TRAPOLD, M. D. (1970). Are expectancies based upon different positive reinforcing events discriminably different? Learning \& Motivation, 1, 129-140.

WICKENS, D. D. (1987). The dual meanings of context: Implications for research, theory, and applications. In D. S. Gorfein \& R. R. Hoffman (Eds.), Memory and learning: The Ebbinghaus Centennial Conference (pp. 135-152). Hillsdale, NJ: Erlbaum

Wickens, D. D., Tuber, D. S., Nield, A. F., Wickens, C. (1977) Memory for the conditioned response: The effects of potential interference introduced before and after original conditioning. Jourmal of Experimental Psychology: General, 106, 47-70.

ZENTALL, T. R. (1970). Effects of context change on forgetting in rats. Journal of Experimental Psychology, 86, 440-448.

(Manuscript received January 10, 1992; revision accepted for publication August 17, 1992.) 